\title{
In this issue...
}

\section{Epigenetic control of beetle mandible size}

Some sexually selected appendages, such as bird tails and beetle horns, display exaggerated sizes and growth rates relative to other body parts, and the augmented growth of such appendages is known to be influenced by nutrition. For example, honeybee larvae raised on nutrient-rich royal jelly develop into queens, whereas larvae fed nutrient-poor worker jelly become workers. Takane Ozawa et al. (pp. 15042-15047) attempted to determine whether epigenetic mechanisms underlie the nutrition sensitivity of sexually selected mandibles in male broad-horned flour beetles (Gnatocerus cornutus). The authors altered the expression levels of genes involved in DNA methylation and histone modification in the beetle larvae through chemical inhibitors and RNA interference. Levels of histone deacetylase (HDAC) enzymes and polycomb family proteins appeared to be associated with

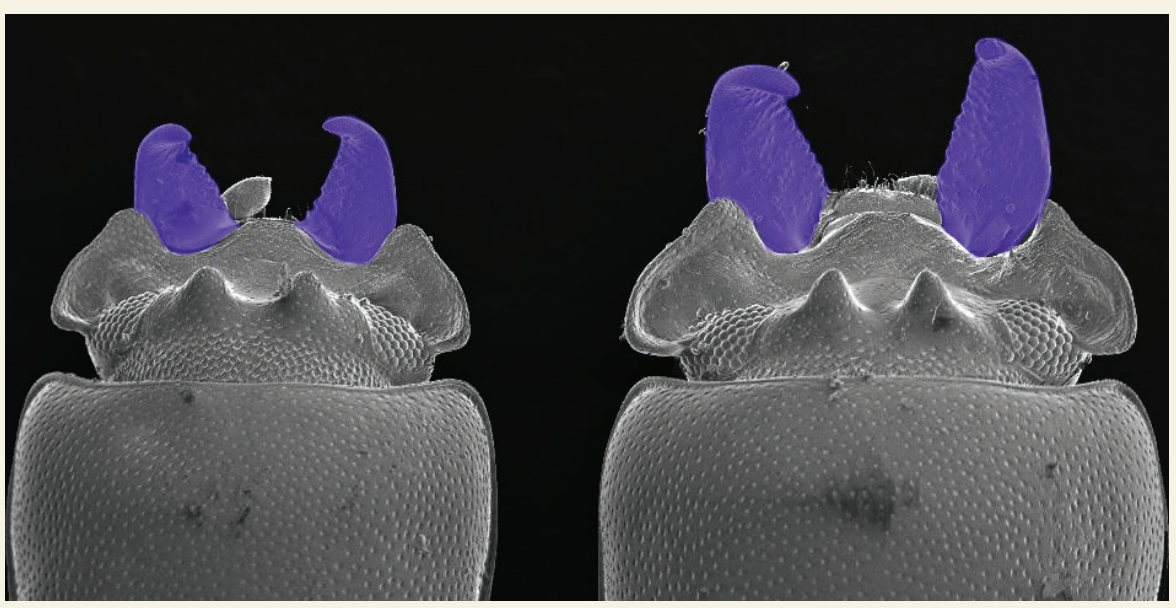

Male mandible polymorphism in broad-horned flour beetle. mandible size, with little effect on core body size or genitalia. Lowering HDAC1 and HDAC3 levels led to shortened and enlarged mandibles in adult beetles, respectively; the interventions had opposite effects on adult wing size, suggesting that the effect may be specific to sexually augmented body parts. Because epigenetic modification is tied to nutrient sensing, the relative sensitivity of sexually selected features to putative nutrition-derived signaling molecules during development might underlie the organ-specific augmentation of such features, according to the authors. - P.N.

\section{Agricultural self-sufficiency in sub-Saharan Africa}

The population of sub-Saharan Africa (SSA) is projected to increase by 2.5 -fold by 2050 , whereas regional cereal consumption may approximately triple by the same year. Food security depends on whether SSA can increase cereal production without increased reliance on cereal imports or major expansion of agricultural area, potentially resulting in increased greenhouse gas emissions and biodiversity loss. To assess agricultural self-sufficiency in SSA, Martin van Ittersum et al. (pp. 14964-14969) used an upscaling approach that incorporates local crop, soil, and weather data to estimate production capacity for the five main cereals across 10 nations in SSA. To maintain the current $80 \%$ level of cereal production self-sufficiency, the region would need to nearly close the gap between current crop yields and crop yield potential on current farmland. Most of the countries

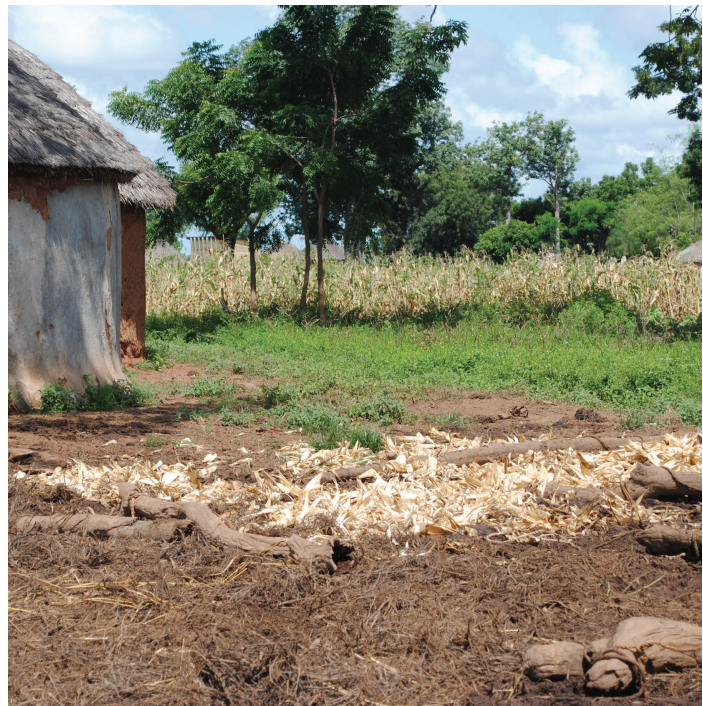

Ghana farm and maize field. 
would require a large, abrupt acceleration in the rate of crop yield gain to meet this goal. The analysis finds that complex and risky techniques for increasing crop yields will be required to reach self-sufficiency. These methods might include increasing the number of crops harvested from a field each year and sustainably expanding irrigated farmland. If SSA cannot quickly improve its yields, the region will likely experience an increased reliance on cereal imports and cropland expansion, according to the authors. - L.C.

\section{Water use and Syrian refugee crisis}

Quantifying the impacts of armed conflict on land and water use has been challenging due to the difficulties of collecting data in active war zones. Marc Müller et al. (pp. 14932-14937) used satellite imagery

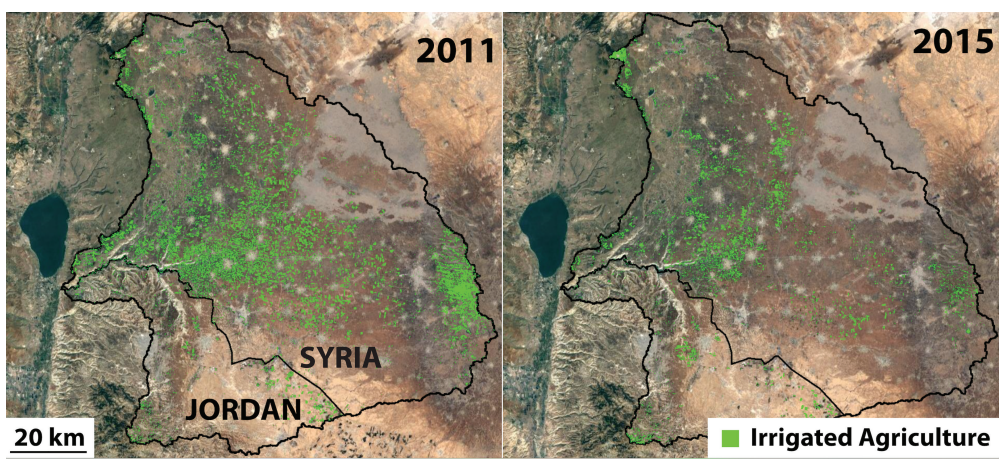

Irrigated land area in the Yarmouk River basin in 2011 (Left) and 2015 (Right). Data courtesy of Landsat 7 (USGS/NASA) and imagery @ 2016 TerraMetrics, map data $\odot 2016$ Google.

to measure irrigated crop area and reservoir storage in the Yarmouk River basin, on the Syria-Jordan border, during the Syrian civil war and consequent refugee migration from the Syrian side of the basin into Jordan. Irrigated area on the Syrian side of the basin declined sharply, compared with the Jordanian side after 2012, coinciding with the flight of the majority of Syrian refugees. Reservoir storage also declined significantly in Syria, compared with the neighboring
Golan Heights of Israel during the same period, suggesting that the flight of refugees caused a significant reduction in farming, and therefore irrigation water use, in Syria. The refugee migration coincided with increased water flow in the Yarmouk River to Jordan. The authors attributed about half of this flow increase to reduced demand for irrigation water and consequent reduced water storage on the Syrian side of the river basin. According to the authors, armed conflict and human displacement may substantially alter the water balance in a river basin, with potential effects on the partitioning of water resources. - B.D.

\section{Childhood poverty and mental health}

A growing body of evidence suggests that childhood poverty is associated with subsequent physical morbidity and mortality. Gary Evans (pp. 14949-14952) investigated whether a similar association exists between childhood poverty and adult psychological health. The author examined mental health symptoms, short-term memory, and indices of helplessness and chronic physiological stress in 341 individuals over a period of 15 years, beginning when the participants were 9 years of age and ending at age 24. The author conducted prospective longitudinal analyses to identify the psychological outcomes at age 24 that could be predicted based on family income relative to the poverty line at age 9, after controlling for baseline differences in psychological variables at age 9 . Family income at age 9 was positively correlated with short-term spatial memory and negatively correlated with helplessness behavior, allostatic load-a measure of chronic physiological stress-and externalizing mental health symptoms, such as aggression at age 24 . Internalizing symptoms, such as depression and anxiety, at age 24 were not significantly correlated with childhood income. The results suggest a link between childhood poverty and reduced psychological well-being in adulthood, according to the author. - B.D. 